Revisiting the Neutral C-Vacancy in Diamond: Localization of Electrons through DFT+U

Peer-reviewed author version

VANPOUCKE, Danny E.P. \& HAENEN, Ken (2017) Revisiting the Neutral C-Vacancy in Diamond: Localization of Electrons through DFT+U. In: DIAMOND AND RELATED MATERIALS, 79, p. 60-69.

DOI: $10.1016 /$ j.diamond.2017.08.009

Handle: http://hdl.handle.net/1942/24894 


\title{
Revisiting the Neutral C-Vacancy in Diamond: Localization of Electrons through $\mathrm{DFT}+\mathrm{U}$
}

\author{
Danny E. P. Vanpoucke $\mathrm{e}^{1,2, *}$ and Ken Haenen ${ }^{1,2}$ \\ ${ }^{1}$ Institute for Materials Research (IMO), Hasselt University, 3590 Diepenbeek, Belgium \\ ${ }^{2}$ IMOMEC, IMEC vzw, 3590 Diepenbeek, Belgium
}

(Dated: August 22, 2017)

\begin{abstract}
The neutral C-vacancy is investigated using density functional theory calculations. We show that local functionals, such as PBE, can predict the correct stability order of the different spin states, and that the success of this prediction is related to the accurate description of the local magnetic configuration. Despite the correct prediction of the stability order, the PBE functional still fails predicting the defect states correctly. Introduction of a fraction of exact exchange, as is done in hybrid functionals such as HSE06, remedies this failure, but at a steep computational cost. Since the defect states are strongly localized, the introduction of additional on-site Coulomb and exchange interactions, through the DFT+U method, is shown to resolve the failure as well, but at a much lower computational cost. In this work, we present optimized U and J parameters for $\mathrm{DFT}+\mathrm{U}$ calculations, allowing for the accurate prediction of defect states in defective diamond. The transferability of the $\mathrm{U}$ and $\mathrm{J}$ parameters is tested through the study of the $\langle 001\rangle$ split-interstitial.
\end{abstract}

Keywords: Diamond, DFT, defect, electronic structure.

\section{INTRODUCTION}

Defects play an important role in the properties and performance of semiconductor devices. Depending on the application, they can change the physical and chemical properties (e.g. the introduction of luminescent centres $^{1,2}$ ) or need to be avoided (e.g. because they deteriorate conductivity ${ }^{3}$ ). In the case of diamond, intrinsic defects have been studied at increasing levels of theory, in lock-step with the advancing state of the art. ${ }^{4-14} \mathrm{In}$ recent years, the use of hybrid functionals has become more and more frequent in the solid state community as a result of ever growing computational resources, and the continuous improvement of methodologies.Some authors even used hybrid functionals in their simulation of infrared and Raman spectra, this of course in combination with small localized basis-sets. ${ }^{15,16}$ Although it is getting quite common to use such hybrid functionals to accurately determine the electronic structure and local spin polarization of complicated systems , ${ }^{17-21}$ their computational cost is still prohibitively high for extensive usage $;^{22}$ e.g. structure optimization of large super cells required for studying single defects. For most structure optimizations, pure density functional theory (DFT) functionals (LDA and GGA) perform admirably well. However, in strongly correlated systems in which the specific electronic structure is tightly related to the atomic structure, problems occur. The neutral C-vacancy in diamond is such a system.

The formation of a neutral vacancy in diamond gives rise to four dangling bonds. This structure has a $T_{d}$ symmetry at this level of consideration. Each dangling bond is occupied by a single electron, which can have either up or down spin. As the other electrons of the $\mathrm{C}$ atoms neighboring the vacancy are involved in strong covalent bonds, the four electrons in the dangling bonds can only couple to each other, giving rise to three possible spin states for the vacancy defect: $S_{z}=0,1$, and 2. The corresponding defect states are expected to be localized at the defect site, with an energy in the band gap of the host material. Of the three spin states only the $S_{z}=2$ state (with all four electrons having the same spin) has the same $T_{d}$ symmetry as the host lattice. In case of the $S_{z}=0$ and 1 spin states, the magnetic configuration has a $D_{2 d}$ and a $C_{3 v}$ symmetry, respectively. In case of the $S_{z}=0$ spin state Jahn-Teller distortion will further lower the symmetry to $C_{2 v} \cdot{ }^{14,23}$ As such, one may expect the modeling of this defect to be problematic.

Recently, Zelferino and co-workers argued that due to the open shell nature of the defect, only functionals including a fraction of the exact exchange term can adequately describe this defect. ${ }^{14}$ They observe that pure DFT functionals present a qualitatively incorrect electronic structure and fail to indicate the correct spin ground state. As vacancies play a crucial role in many technologically interesting defects in diamond, such as the NV-center ${ }^{24-27}$ it is important to understand how and why pure DFT fails for the vacancy defect. Furthermore, as the solution of hybrid functionals is too computationally expensive for large scale usage, an alternative post-DFT approach is desirable, albeit only as a means of providing a more accurate defect geometry for subsequent analysis using a hybrid functional.

In this work, we will therefore revisit the neutral $\mathrm{C}$ vacancy in diamond. After introducing the computational methods used, results are presented and discussed in section 3. We first examine the behavior of the defect using a pure DFT functional, and show how using knowledge of the magnetic configuration can help us avoid getting stuck in a local minimum. The qualitative failure with regard to the defect states is recognised as the well-known band gap problem of DFT. Hybrid functional calculations are performed to 
provide reference data, and subsequently we present a $\mathrm{DFT}+\mathrm{U}$ study of the defect. We show how the on-site Coulomb and exchange parameters, U and J, influence different parts of the electronic structure independently. An optimum value of $(\mathrm{U}, \mathrm{J})=(8.56,15.06) \mathrm{eV}$ is obtained after fitting to the reference hybrid functional electronic structure obtained. After validating the (U,J)-pair for the neutral vacancy defect, we also address the $\langle 001\rangle$ split-interstitial defect to test the transferability of the $\mathrm{U}$ and $\mathrm{J}$ parameters. The conclusions are presented in the final section of the manuscript.

\section{COMPUTATIONAL DETAILS}

The single vacancy defect is simulated using a conventional cubic 64-atom cell from which a single $\mathrm{C}$ atom is removed (see Fig. 1), giving rise to a vacancy concentration of $1.56 \%$. Although vacancy concentrations are generally lower in experiments, a super cell of this size gives a reasonable qualitative picture of the electronic structure. As the goal of the present work is to provide a computationally cheaper alternative to hybrid functional calculations, rather than a quantitative study of the neutral vacancy defect itself, this super cell suffices for our needs. DFT calculations are performed within the projector augmented wave (PAW) method as implemented within the Vienna $a b$ initio Simulation Package (VASP). Because of the expected gradients in the electron density around the vacancy defect, the exchange and correlation behaviour of the valence electrons is described using the generalised gradient approximation (GGA) as constructed by Perdew, Burke and Ernzerhof (PBE). In addition, high quality reference electronic structures are calculated using the HSE06 hybrid functional. This hybrid functional is specifically designed for solids, and has been used successfully for materials ranging from (porous) Metal-Organic Frameworks to dense solids like diamond. ${ }^{18,28-30}$ The structure is optimized using the PBE functional. During structure optimisation lattice parameters and ionic positions are allowed to optimise simultaneously using a conjugate gradient method. An energy-convergence criterion is used, which is set to $1.0 \times 10^{-7}$. After the full relaxation, forces on the ions are found to be below $0.5 \mathrm{meV} / \mathrm{A}$ for all systems but one, for which the largest force is found to be $1.12 \mathrm{meV} / \mathrm{A}$. For all calculations the kinetic energy cutoff is set to $600 \mathrm{eV}$. The first Brillouin zone is sampled using a MonkhorstPack special $k$-point grid of $4 \times 4 \times 4$ (PBE relaxation and HSE06 post-processing calculations), $5 \times 5 \times 5(\mathrm{PBE}$ static and frequency calculations) or $9 \times 9 \times 9$ (PBE density of states) $k$-points.

The $4 \times 4 \times 4 \mathrm{k}$-point grid for the HSE06 calculations is checked to be well converged compared to the $5 \times 5 \times 5$ $\mathrm{k}$-point grid, and 4 times faster due to the reduction in number of k-points (change in energy of the system was of the order of $0.01 \mathrm{meV}$ for the 64 atom cell). In ad- dition, we check if the k-point grid for the Hartree-Fock part of the functional could be further reduced. Halving it to a $2 \times 2 \times 2 \mathrm{k}$-point grid (combined with the $4 \times 4 \times 4 \mathrm{k}$-point grid for the local part of the functional) reduced the accuracy to about $5 \mathrm{meV}$ for the 64 atom cell, while a reduction to the $\Gamma$-point gives rise to an energy difference of nearly $2 \mathrm{eV}$, making the latter settings entirely unacceptable. In addition, a $6 \times 6 \times 6 \mathrm{k}$-point grid (for the local part of the functional) combined with a $2 \times$ and $3 \times$ reduced $\mathrm{k}$-point grid for the non-local part of the functional is considered. The accuracy of the energy is comparable to that found for the $4 \times 4 \times 4 \mathrm{k}$-point grid above, although at a higher computational cost. As a result, considering the computational cost, accuracy, and number of required hybrid calculations for this work, we choose to use a $4 \times 4 \times 4 \mathrm{k}$-point grid for both the local and non-local part of the HSE06 functional.
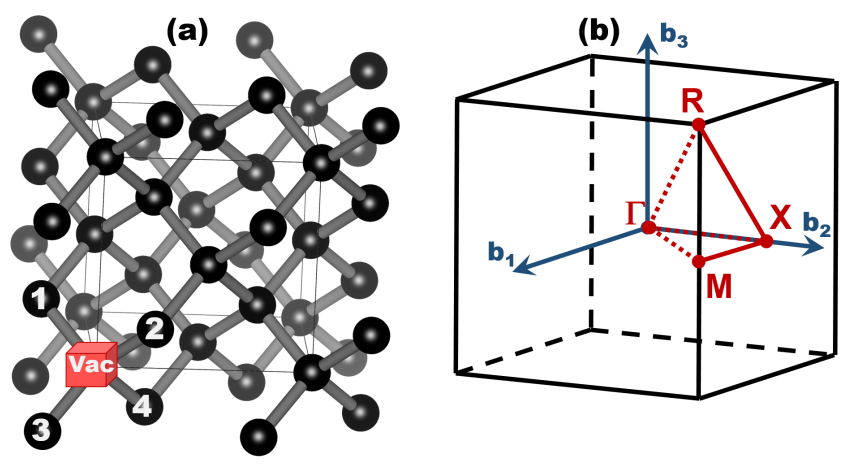

FIG. 1. (a) Ball-and-stick representation of a single Cvacancy defect (red cube) in a cubic diamond lattice. The conventional cubic cell is indicated by the black lines. The four neighbouring $\mathrm{C}$ atoms are indicated. (b) High symmetry lines of the first Brillouin zone of a simple cubic system.

\section{RESULTS AND DISCUSSION}

The neutral $\mathrm{C}$ vacancy defect has previously been studied both theoretically and experimentally by several researchers. ${ }^{4-14,23,31}$ The experimental picture has clearly converged showing a diamagnetic ground state $\left(S_{z}=0\right)$, with the $S_{z}=1$ state being slightly less stable, and the high spin $S_{z}=2$ state much less stable. The theoretical picture for DFT calculations appear less coherent with conflicting results regarding the actual ground state spin configuration. To understand the source of these discrepancies we start by revisiting the neutral $\mathrm{C}$ vacancy in the standard DFT case. In the following subsection we will also present results obtained with the hybrid functional HSE06 which will be used as reference later on. 


\section{A. Revisiting the C-vacancy using DFT and hybrid functional}

\section{PBE without symmetry breaking}

The initial geometry for the $\mathrm{C}$ vacancy defect is created by removing a single $\mathrm{C}$ atom from a cubic 64 -atom super cell with a perfect diamond lattice. The structure is subsequently optimized with the only constraint being the difference in number of electrons with up $(\uparrow)$ and down $(\downarrow)$ spin, $\Delta_{\uparrow, \downarrow}$ for the entire cell. Within this work we will assume systems for which $\Delta_{\uparrow, \downarrow}=0,2$, and 4 identify with the spin state $S_{z}=0,1$, and 2 , respectively, and therefore use the spin states nomenclature to refer to the different systems. After structure optimization, the lattice parameter is found to be increased by 0.05 , 0.04 and $0.24 \%$ for the $S_{z}=0,1$, and 2 systems, respectively, which is to be expected with the introduction of a vacancy. ${ }^{32}$

The vacancy formation energy is calculated as:

$$
E_{f}=E_{\text {def }}-N_{C} \mu_{C},
$$

with $E_{\text {def }}$ the total energy of the super cell with the defect, $N_{C}$ the number of $\mathrm{C}$ atoms in the super cell with the defect, and $\mu_{C}$ the chemical potential of a $\mathrm{C}$ atom, chosen to be the bulk energy for $\mathrm{C}$ in the diamond structure. In contrast to experiment, the vacancy formation energies presented in Table I identify the $S_{z}=1$ state as the most stable configuration. This is, however, in line with results presented in theoretical literature for super cells containing 32, 64, or 128 atoms. ${ }^{8,14}$ For each of the spin states the same $\mathrm{T}_{d}$ space group is found, in contrast to what is expected from a molecular-like model. ${ }^{23}$ Investigation of the normal-modes presents, in case of the $S_{z}=0$ system, only three small imaginary frequencies with energies $<0.2 \mathrm{meV}$ (about two orders of magnitude smaller than the smallest real frequencies), representing the zero-modes linked to the three translational degrees of freedom. In case of the $S_{z}=1$ system two additional imaginary frequencies are found, which means this system resides in a transition state. The total energy of the 5 imaginary frequencies is about $5.5 \mathrm{meV}$ which is still rather small. For the $S_{z}=2$ system, also three imaginary frequencies are obtained, which are at least one order of magnitude smaller than what was found for the $S_{z}=0$ system. This presents an image of reasonably well converged structures, although comparison to experimental data tells us this is not the case.

To further investigate these systems we consider the magnetisation of the four $\mathrm{C}$ atoms neighbouring the vacancy site, given in Table II. The presented magnetisation is obtained through the integration of the magnetic moment inside the PAW sphere. For the $S_{z}=2$ system, the magnetisation of the four neighbouring $\mathrm{C}$ atoms is the same in size and sign, in line with what we expect for four electrons in the highest spin configuration. In contrast, the $S_{z}=0$ system shows
TABLE I. Properties of C-vacancy systems

\begin{tabular}{|c|c|c|c|c|}
\hline & $\begin{array}{r}E_{f} \\
(\mathrm{eV})\end{array}$ & $\begin{array}{r}\Delta_{0, i} \\
(\mathrm{meV})\end{array}$ & Symm. & $\begin{array}{l}\mathrm{ZPE} \\
(\mathrm{eV})\end{array}$ \\
\hline & \multicolumn{4}{|c|}{$\mathrm{PBE}$} \\
\hline$\overline{S_{z}=0}$ & 6.659 & 0 & $\mathrm{~T}_{d}$ & 10.962 \\
\hline$S_{z}=1$ & 6.521 & -138 & $\mathrm{~T}_{d}$ & 10.944 \\
\hline \multirow[t]{2}{*}{$\underline{S_{z}=2}$} & 8.156 & 1497 & $\mathrm{~T}_{d}$ & 11.026 \\
\hline & \multicolumn{4}{|c|}{ PBE symm.br. } \\
\hline$\overline{S_{z}}=0$ & 6.443 & 0 & $\mathrm{D}_{2 d}$ & 11.062 \\
\hline$S_{z}=1$ & 6.522 & 79 & $\mathrm{C}_{3 v}$ & 10.947 \\
\hline \multirow[t]{2}{*}{$\underline{S_{z}=2}$} & 8.139 & 1696 & $\mathrm{~T}_{d}$ & 11.026 \\
\hline & \multicolumn{4}{|c|}{ HSE06 } \\
\hline$\overline{S_{z}}=0$ & 7.009 & 0 & $\mathrm{D}_{2 d}$ & - \\
\hline$S_{z}=1$ & 7.149 & 141 & $\mathrm{C}_{3 v}$ & - \\
\hline \multirow[t]{2}{*}{$S_{z}=2$} & 8.469 & 1461 & $\mathrm{~T}_{d}$ & - \\
\hline & \multicolumn{4}{|c|}{$\mathrm{PBE}+\mathrm{U}$} \\
\hline$\overline{S_{z}}=0$ & 6.289 & 0 & $\mathrm{D}_{2 d}$ & 12.309 \\
\hline$S_{z}=1$ & 6.442 & 153 & $\mathrm{C}_{3 v}$ & 12.307 \\
\hline$S_{z}=2$ & 7.385 & 1096 & $\mathrm{~T}_{d}$ & 12.286 \\
\hline
\end{tabular}

no net magnetisation on any of the four neighboring $\mathrm{C}$ atoms. This clearly shows that although we have the expected global magnetisation, this system clearly does not reside in the $S_{z}=0$ spin state. Similarly we find that also the $S_{z}=1$ system did not actually produce the required $S_{z}=1$ spin state.

Although the global magnetization may indicate the systems to be in the $S_{z}=0,1$, or 2 spin state and the normal-modes present reasonable quality structures, the local magnetization of the neighboring $\mathrm{C}$ atoms shows that the true $S_{z}=0,1$, or 2 spin states are not obtained. This explains the erroneous stability ordering and local symmetry.

At this point it is important to note that using these obtained atomic structures, and using the same constraints, hybrid functionals will not improve the situation. Optimizing the electronic structure using the HSE06 functional, the same erroneous stability ordering is found (although this time $\Delta_{0,1}=-650 \mathrm{meV}$ ) as well as the same local magnetization as was found for the PBE calculations. From this it becomes clear that the obtained systems are local energy minima, albeit not the ones intended.

\section{PBE with symmetry breaking}

As the problem of determining the correct states seems to be hampered by the presence of similar local minima, the obvious way forward is to provide starting conditions closer to the intended spin states. Starting from the same initial geometry as before (pure diamond lattice with a single $\mathrm{C}$ atom removed), including the same constraint on the global magnetization, we now initialize the local magnetization of the four $\mathrm{C}$ atoms neighboring the vacancy in line with their intended spin 
TABLE II. Magnetisation of four vacancy neighbour carbon atoms.

\begin{tabular}{l|rrrr|rrrrrrrr}
\hline \hline & \multicolumn{9}{|c}{$S_{z}=0$} & \multicolumn{4}{c}{$S_{z}=1$} & \multicolumn{4}{c}{$S_{z}=2$} \\
functional & $\mathrm{C}_{1}$ & $\mathrm{C}_{2}$ & $\mathrm{C}_{3}$ & $\mathrm{C}_{4}$ & $\mathrm{C}_{1}$ & $\mathrm{C}_{2}$ & $\mathrm{C}_{3}$ & $\mathrm{C}_{4}$ & $\mathrm{C}_{1}$ & $\mathrm{C}_{2}$ & $\mathrm{C}_{3}$ & $\mathrm{C}_{4}$ \\
\hline PBE & 0.000 & 0.000 & 0.000 & 0.000 & 0.366 & 0.327 & 0.217 & 0.212 & 0.485 & 0.487 & 0.488 & 0.487 \\
PBE symm.br. & 0.366 & -0.330 & 0.288 & -0.325 & 0.159 & 0.300 & 0.317 & 0.341 & 0.486 & 0.489 & 0.489 & 0.489 \\
HSE06 & 0.424 & -0.410 & 0.392 & -0.409 & -0.232 & 0.449 & 0.446 & 0.452 & 0.514 & 0.518 & 0.518 & 0.518 \\
PBE+U & 0.372 & -0.365 & 0.350 & -0.361 & -0.267 & 0.385 & 0.394 & 0.395 & 0.429 & 0.431 & 0.432 & 0.432 \\
\hline \hline
\end{tabular}

for three different spin states. This has the consequence that the spin symmetry is broken even though the atomic starting geometry remains $\mathrm{T}_{d}$. We indicate these systems as "PBE symm.br." in the tables.

After structure optimization, the lattice parameters are found to have increased by $0.20,0.04$, and $0.24 \%$ for the $S_{z}=0,1$, and 2 spin state, respectively. In case of the $S_{z}=0$ system, the lattice is no longer cubic, but has become tetragonal with a single direction shortened by $0.22 \%$ in comparison to the cubic diamond lattice. The calculated vacancy formation energy ( $c f$. Table I) shows a significant reduction for the $S_{z}=0$ system resulting in the experimentally expected singlet ground state. The relative vacancy formation energy is well in line with the value $0.06 \mathrm{eV}$ obtained by Zywietz and co-workers for a 216-atom super cell, as well as the $\mathrm{D}_{2 d}$ symmetry. ${ }^{9}$ Note that the $\mathrm{C}_{2 v}$ space group is a direct subgroup of the $\mathrm{D}_{2 d}$ space group. The $S_{z}=1$ system did not change in energy even though the symmetry changed significantly, from the $\mathrm{T}_{d}$ to the $\mathrm{C}_{3 v}$ space group. For all systems, investigation of the normal-modes showed only three (small) imaginary frequencies, indicative of a local minimum.

In addition, investigation of the local magnetization, presents a significant improvement over the results presented in the previous section. The $S_{z}=0$ system clearly shows two $\mathrm{C}$ atoms with a localized up-spin electron, and two with a localized down-spin electron. The $S_{z}=1$ system, on the other hand presents three $\mathrm{C}$ atoms with a 'large' up-spin magnetization, and one $\mathrm{C}$ atom with a 'smaller' magnetization, unfortunately still with the wrong spin orientation, but already a qualitative improvement.

\section{HSE06 reference electronic structure}

Starting from the optimized atomic structures in the previous section (PBE symm.br.), which show the required symmetry breaking, the electronic structure of the systems can be optimized at the hybrid functional level.

The vacancy formation energy increases by about 0.6 $\mathrm{eV}$ for the $S_{z}=0$ and $S_{z}=1$ systems and $0.3 \mathrm{eV}$ for the $S_{z}=2$ system, retaining the experimental stability order. The local magnetization shows the correct qualitative picture for all three spin states, in contrast to all previous PBE calculations.

For the PBE calculations, the electronic band structure along the high symmetry lines (see Fig. 1b) are shown in Fig. 2. For each system, the defect states can be clearly differentiated in the band gap of pristine diamond. Furthermore, comparison to the HSE06 band structure, shown in Fig. 3, shows that the PBE calculations are able to reproduce the qualitative picture to a reasonable degree. The shape of the bands is well represented, while their main failure is their position, which is due to the well-known band gap problem of DFT. The obtained HSE06 electronic band structure is in good agreement with previous theoretical work, presenting band gap sizes as well as the defect band locations as expected. ${ }^{14}$

Although most of the defect bands show the correct qualitative picture some states show significant deviations (those bands are indicated in green in Fig. 2). The $S_{z}=0$ system seems to be reproduced best by $\mathrm{PBE}$, with only a part of the middle defect band along the $\mathrm{R}-\Gamma$ line showing the up and down state not coinciding as seen for the HSE06 case. The $S_{z}=1$ system shows a significant split between the middle defect bands (the top up band and the bottom down band) leading to the observed metallic behavior of this spin state in PBE. In case of the $S_{z}=2$ spin state, the main problem is the location of the bottom spin down defect state, which should be located at the Fermi level together with the spin down defect states but is located more than $1 \mathrm{eV}$ below the Fermi level.

\section{B. The C-vacancy using DFT+U}

Although hybrid functional theory calculations are becoming increasingly popular for solid state applications, their computational cost is still staggering. For the presented systems, we find an increase in computational cost of about a factor one thousand going from PBE to HSE06 calculations while retaining the same accuracy settings. ${ }^{22}$

An alternate means of improving the electronic structure for solids in case of strongly correlated systems or electronic structures dealing with strongly localized states is the DFT+U approach. The basic idea behind this methodology is to correct the on-site Coulomb interaction of the localized electrons by adding Hubbard-like terms. Although these corrections are usually included 

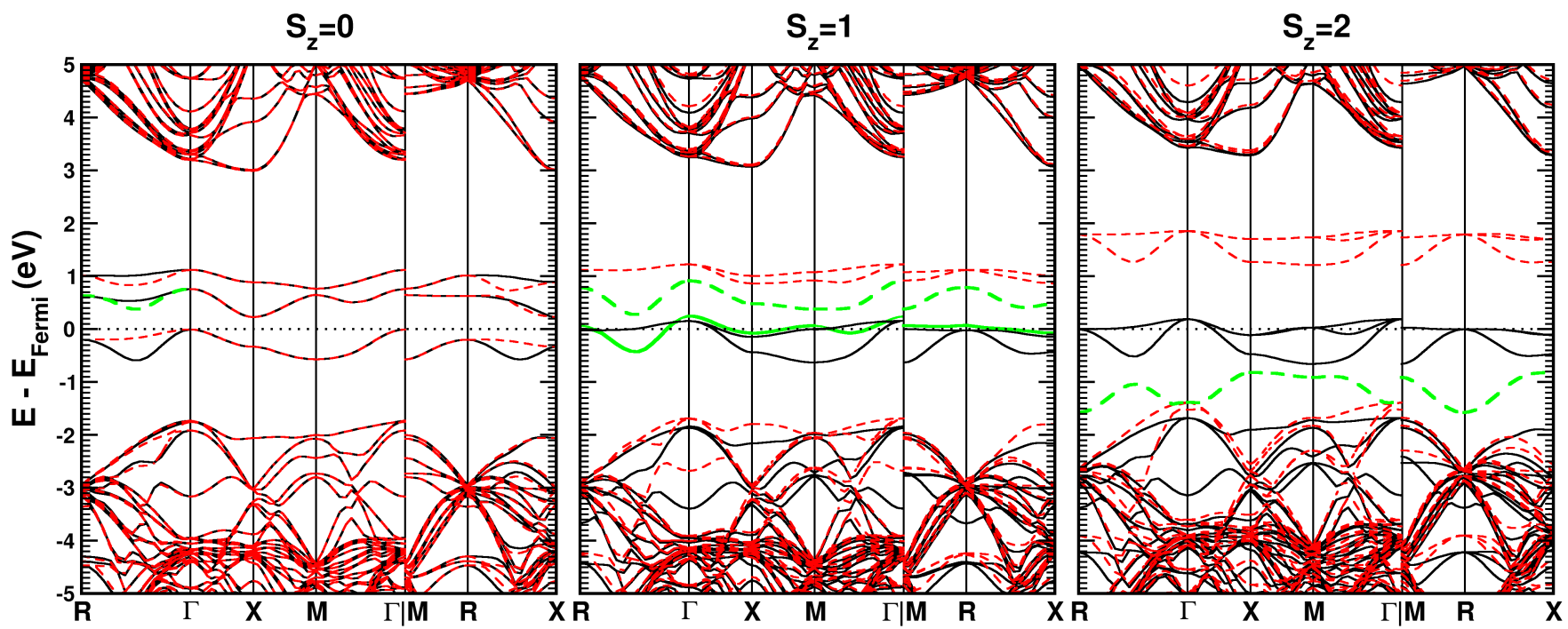

FIG. 2. PBE electronic band structure for a single $\mathrm{C}$ vacancy defect in a 64-atom super cell. Majority/minority bands are show as solid/dashed curves. Bands indicated in green are qualitatively different from the HSE06 picture.


FIG. 3. HSE06 electronic band structure for a single $\mathrm{C}$ vacancy defect in a 64-atom super cell. Fitting variables for the DFT+U fitting procedure are indicated.

when dealing with $d$ or $f$ electrons, they can also be beneficial for localized $p$ orbitals, ${ }^{33}$ as we will show below. There exist different ways to include these corrections in DFT calculations. In this work we make use of the rotationally invariant $\mathrm{DFT}+\mathrm{U}$ implementation as proposed by Liechtenstein and co-workers which makes use of two parameters: $\mathrm{U}$ and $\mathrm{J} .{ }^{34}$ The choice of this approach is based on the fact that for the system at hand U and J are independent parameters, and need to be treated as such. This is not the case for the simplified DFT $+\mathrm{U}$ approach as proposed by Dudarev and co-workers, in which $\mathrm{U}$ and $\mathrm{J}$ are combined into a single $\mathrm{U}_{\text {eff }}=\mathrm{U}-\mathrm{J} .{ }^{35}$ From Fig. 4, showing the example of the $S_{z}=1$ system, it is clear that such a simplified approach should not be used for defective diamond. Even though $\mathrm{U}_{\text {eff }}$ is constant, the defect state positions and shapes are changing significantly, while the direct band gap of the host system (pristine diamond) remains roughly the same over the entire range.

Figures 5 and 6 show the evolution of the electronic band structure of the $S_{z}=1$ system at constant J and $\mathrm{U}$, respectively. Increase of the on-site Coulomb parameter $(\mathrm{U})$ gives rise to a reduction of the band gap of the diamond host, while at the same time pulling the defect states open. The unoccupied down-states remain unoccupied with the upper band even moving into the conduction band. The three up-states, which give rise to the metallic nature under PBE, also split, making the system 

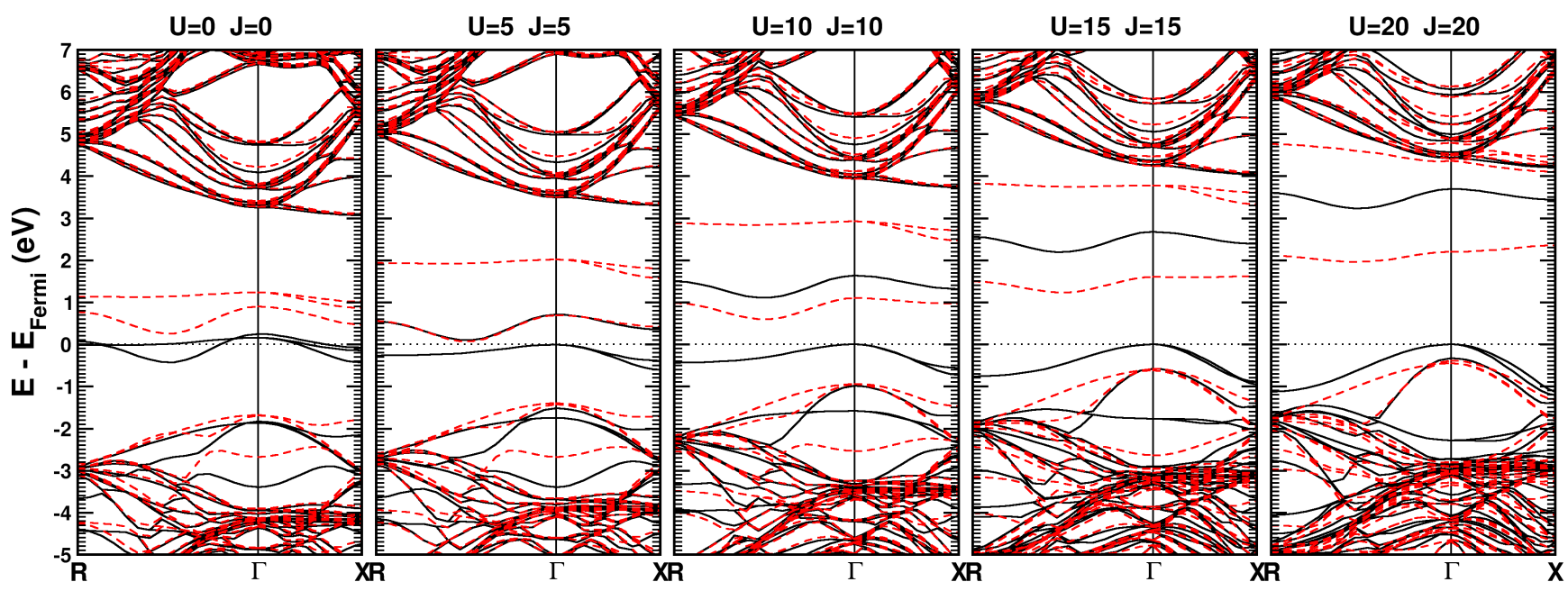

FIG. 4. Evolution of the electronic band structure as function of $\mathrm{U}$ and $\mathrm{J}$, with $\mathrm{U}=\mathrm{J}$ or $\mathrm{U}_{\text {eff }}=0 \mathrm{eV}$.
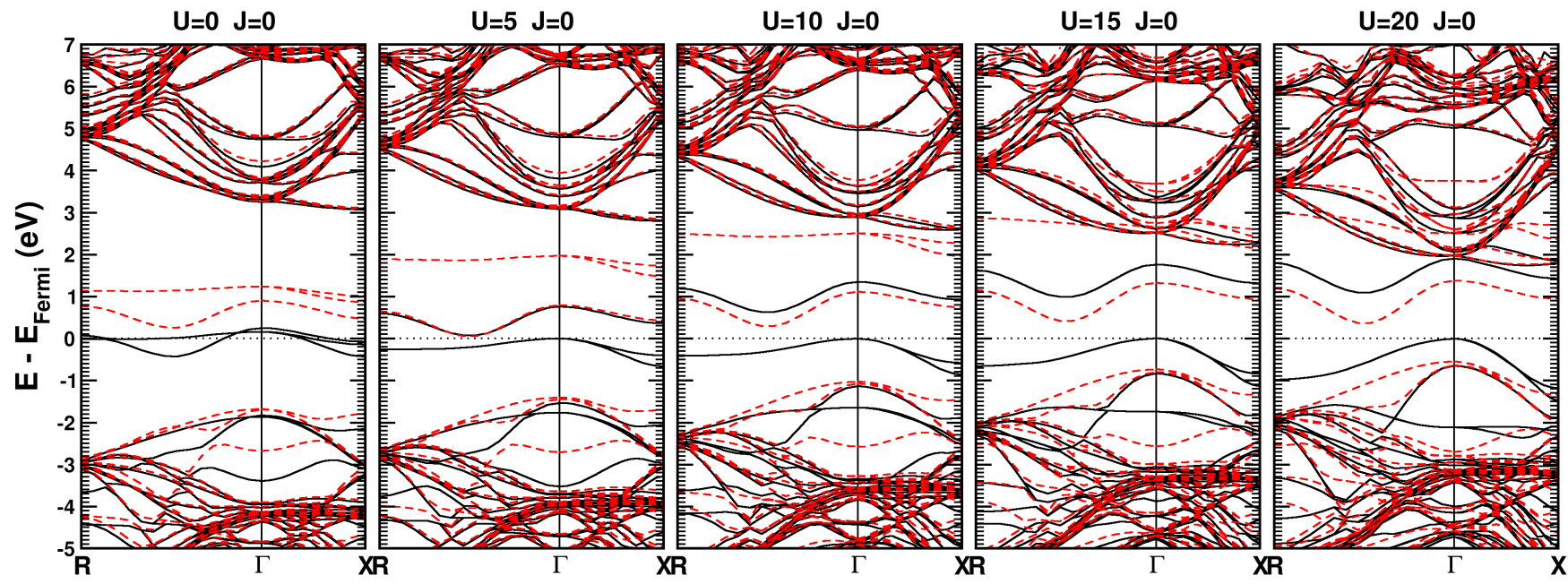

FIG. 5. Evolution of the electronic band structure as function of $\mathrm{U}$, for $\mathrm{J}=0 \mathrm{eV}$.

semiconducting for $\mathrm{U} \geq 5 \mathrm{eV}$.

In contrast, keeping $\mathrm{U}$ constant while increasing the onsite exchange parameter $\mathrm{J}$, only slightly compresses the defect states while opening up the band gap of the diamond host (see Fig. 6). This opening of the band gap has been observed for negative $\mathrm{U}_{\text {eff }}$ in other $s p$-hybridized semiconductors as well, and is related to the localization of the $p$-states making up the valence band edge. ${ }^{33}$

\section{Fitting $U$ and $J$ parameters}

Since the on-site Coulomb and exchange parameters are independent, we fit both. Because it is our goal to reproduce the HSE06 electronic structure as accurately as possible, we use the HSE06 results for the three spin states as a reference. The 14 selected variables, chosen to represent specific features of the electronic structure, are shown in Table III and indicated in Fig 3. Because the direct band gap at the $\Gamma$ of the host diamond is overestimated by HSE06 (due to the introduced exact exchange) we decided do use the experimental band gap of $5.47 \mathrm{eV}$ instead during the fitting procedure.

Fitting is done using a least squares method, minimizing

$$
S(U, J)=\sum_{i} w_{i}\left(x_{i}(U, J)-x_{i}^{\mathrm{ref}}\right)^{2}
$$

where the sum over $i$ runs over a (sub)set of fitting variables, $x_{i}^{\text {ref }}$ is the HSE06 reference value, $x_{i}(U, J)$ is the DFT $+\mathrm{U}$ calculated value of variable $x_{i}$ using the on-site Coulomb and exchange parameters $\mathrm{U}$ and $\mathrm{J}$, and $w_{i}$ a weighing function. The most simple version of $w_{i}$ gives the same weight of 1.0 to all variables (indicated as w/o weight in Table IV). However, as the range of possible values for a given variable can differs strongly 

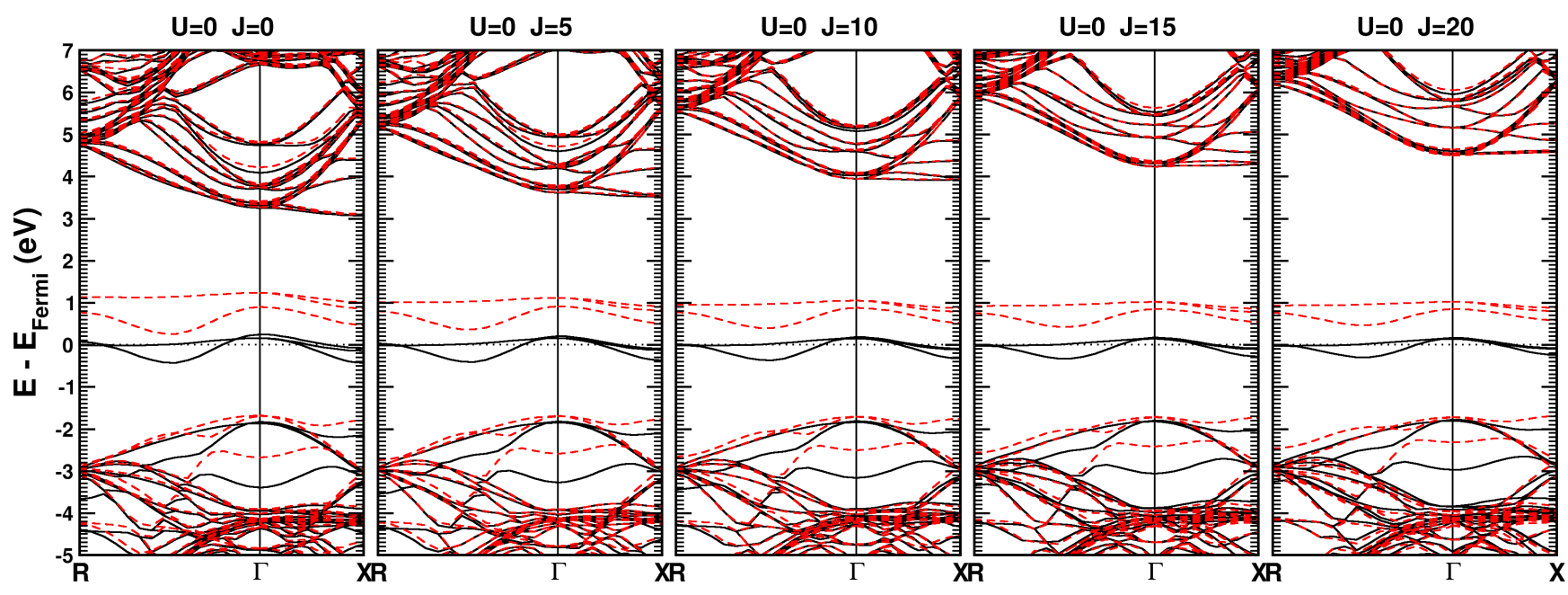

FIG. 6. Evolution of the electronic band structure as function of $\mathrm{J}$, for $\mathrm{U}=0 \mathrm{eV}$.

TABLE III. Fitting variables

\begin{tabular}{|c|c|c|}
\hline & \multicolumn{2}{|c|}{$\begin{array}{c}\text { variable Ref.Value description } \\
(\mathrm{eV})\end{array}$} \\
\hline & \multicolumn{2}{|c|}{ general } \\
\hline$\overline{1}$ & $\Delta_{0,1}$ & 0.141 relative stability of $S_{z}=1$ \\
\hline \multirow[t]{2}{*}{2} & $\Delta_{0,2}$ & 1.461 relative stability of $S_{z}=2$ \\
\hline & & $S_{z}=0$ spin state \\
\hline 3 & $\Delta^{\Gamma, 0}$ & $6.128^{a}$ direct band gap of host for $\uparrow$ at $\Gamma$ \\
\hline 4 & $\Delta_{1,2}^{\Gamma, 0}$ & -2.044 gap between $\uparrow$ states 1 and 2 at $\Gamma$ \\
\hline 5 & $\Delta_{1,3}^{\Gamma, 0}$ & -2.813 gap between $\uparrow$ states 1 and 3 at $\Gamma$ \\
\hline \multirow[t]{2}{*}{6} & $\Delta_{2,2^{*}}^{R^{\prime}, 0}$ & $\begin{array}{c}0.000 \text { coinciding of second defect state } \\
\uparrow \text { and } \downarrow \text { at middle of } R-\Gamma \text { line }\end{array}$ \\
\hline & & $S_{z}=1$ spin state \\
\hline$\overline{7}$ & $\Delta^{\Gamma, 1}$ & $6.221^{a}$ direct band gap of host for $\uparrow$ at $\Gamma$ \\
\hline 8 & $\Delta_{1,3}^{\Gamma, 1}$ & -1.691 gap between $\uparrow$ states 1 and 3 at $\Gamma$ \\
\hline \multirow[t]{2}{*}{9} & $\Delta_{3,1^{*}}^{R^{\prime}, 1}$ & $\begin{array}{l}0.185 \text { splitting between third } \uparrow \text { and first } \downarrow \\
\text { state at middle of } R-\Gamma \text { line }\end{array}$ \\
\hline & & $S_{z}=0$ spin state \\
\hline 10 & $\Delta^{\Gamma, 2}$ & $6.548^{a}$ direct band gap of host for $\uparrow$ at $\Gamma$ \\
\hline 11 & $\Delta_{1,1^{*}}^{\Gamma, 2}$ & -3.366 gap between first $\uparrow$ and $\downarrow$ state at $\Gamma$ \\
\hline 12 & $\Delta_{1,0^{*}}^{\Gamma, 2}$ & 0.340 cross-splitting at $\Gamma$ \\
\hline 13 & $\Delta_{1,0^{*}}^{R^{\prime}}$ & -0.896 cross-splitting at middle of $R-\Gamma$ line \\
\hline 14 & $\Delta_{3,0^{*}}^{R^{\prime}, 2}$ & -0.264 cross-splitting at middle of $R-\Gamma$ line \\
\hline
\end{tabular}

between the variables we also used a weighing variable $w_{i}=a b s\left(\max \left(x_{i}(U, J)\right)-\min \left(x_{i}(U, J)\right)\right.$. In this case, a strongly varying variable will carry a larger weight than a variable which only varies very little over the entire set of (U,J) pairs. The RMSD for the fit is calculated as $\mathrm{RMSD}=\sqrt{S(U, J) / n}$ with $\mathrm{n}$ the number of fitting variables considered.

The $x_{i}(U, J)$ variables are obtained for $\mathrm{U}$ and $\mathrm{J}$
TABLE IV. Fitted U and J parameters (in eV).

\begin{tabular}{l|rrrrrr}
\hline \hline \multirow{2}{*}{$\begin{array}{c}\text { variables } \\
\text { used }\end{array}$} & \multicolumn{3}{|c}{ w/o weight } & \multicolumn{3}{c}{ with weight } \\
\hline 1,2 & 3.71 & 5.49 & 0.00 & 3.71 & 5.49 & 0.00 \\
$1,2,3,7,10$ & 3.79 & 9.68 & 0.10 & 3.79 & 10.02 & 0.17 \\
$3-6$ & 8.67 & 17.89 & 0.08 & 8.82 & 17.78 & 0.13 \\
$7-9$ & 8.22 & 16.61 & 0.17 & 8.44 & 16.77 & 0.24 \\
$10-14$ & 10.51 & 9.51 & 0.11 & 10.36 & 9.63 & 0.19 \\
$3-14$ & 8.82 & 14.51 & 0.20 & 8.73 & 15.09 & 0.31 \\
$4-6,8,9,11-14$ & 9.24 & 13.55 & 0.16 & 9.52 & 12.68 & 0.24 \\
$1-14$ & 8.57 & 15.04 & 0.20 & 8.56 & 15.45 & 0.32 \\
\hline \hline
\end{tabular}

varying from 0 to $20 \mathrm{eV}$ in steps of $1 \mathrm{eV}$ using the atomic structure as obtained in the PBE calculations. Values at non-integer $(\mathrm{U}, \mathrm{J})$-pairs are obtained through bilinear interpolation.

The results of fitting, taking into account several subsets of the 14 variables, are shown in Table IV. Contrary to expectations, the weighing factors seem to play only a minor role in determining the fitted $(\mathrm{U}, \mathrm{J})$-pair. Taking only the relative stabilities of the three spin systems, a perfect fit to the HSE06 values is obtained for $(\mathrm{U}, \mathrm{J})=(3.71,5.49) \mathrm{eV}$. However, at this value for $(\mathrm{U}, \mathrm{J})$, the electronic structure does not show the qualitative and quantitative agreement aimed for. Including the direct band gap at the $\Gamma$-point leads to a significant increase of the $\mathrm{J}$ parameter, as this parameter is the one responsible for opening the host band gap as is seen from Fig. 6. At this point it is important to note that using the experimental band gap at $0 \mathrm{~K}(5.4$ $\mathrm{eV})$ or room temperature $(5.47 \mathrm{eV})$ does not modify the obtained $(\mathrm{U}, \mathrm{J})$ pair significantly. Fitting the $(\mathrm{U}, \mathrm{J})$-pair for the specific electronic features of the three spin states independently shows comparable results for the $S_{z}=0$ and $S_{z}=1$ spin states, while the $S_{z}=2$ spin state is the only setup in which $U>J$. This is related to the lowest of the four $\downarrow$ defect states, which is located at the 
diamond valence band edge for the $S_{z}=0$ and $S_{z}=1$ spin states, while it is pulled up to the Fermi level for the $S_{z}=2$ spin state ( $c f$. Fig. 3). Taking the electronic structure variables into account of all three spin states $(3-14)$, the fitted $(\mathrm{U}, \mathrm{J})$-pair is found to be about $(9,15)$ $\mathrm{eV}$, reducing the difference between $\mathrm{U}$ and $\mathrm{J}$ compared to the fitting obtained for the $S_{z}=0$ and $S_{z}=1$ variables separately. Furthermore, taking this set of 12 variables and excluding the direct band gap variables further reduces the separation between $\mathrm{U}$ and $\mathrm{J}$, again showing the opening of the band gap in $s p$-hybridized semiconductors to be governed by a delocalization of the $p$ states of the valence band. ${ }^{33}$ Taking the full set of 14 variables into account, we find value ranges of $\mathrm{U}=8.56-8.57 \mathrm{eV}$ and $\mathrm{J}=15.04-15.45 \mathrm{eV}$.

\section{The neutral $C$-vacancy}

Using the DFT+U fitting parameters $(\mathrm{U}, \mathrm{J})=(8.56,15.06) \mathrm{eV}$, we perform a full structure optimization of the three spin-states. Allowing for volume relaxation, we find that the volume of the system decreases by $7.1-7.3 \%$ making the lattice parameter obtained for pure diamond at $\mathrm{PBE}+\mathrm{U}$ about $2.4 \%$ too small, in comparison a near perfect match for $\mathrm{PBE}$ $(0.08 \%$ too big). After structure optimization, under constraints setting the total global magnetization and initializing the local magnetization, the correct stability order is found (see Table I). The defect formation energies are 154, 80, and $754 \mathrm{meV}$ lower than those found with $\mathrm{PBE}$ for the $S_{z}=0,1$, and 2 spin states, respectively. The resulting defect formation energies for $\mathrm{PBE}+\mathrm{U}$, presented in Table I, still nicely fall in the range of defect formation energies for the neutral C-vacancy $(5.95-7.65 \mathrm{eV})$ found in the theoretical literature of the past 30 years, making the $\mathrm{PBE}+\mathrm{U}$ values not unacceptably small. , $^{5,10-14}$

As before, we find a broken symmetry for the $S_{z}=0$ and $S_{z}=1$ system, and a normal mode analysis shows only three small imaginary frequencies $(<0.02 \mathrm{meV})$, representing the translational degrees of freedom, for all three spin configurations. The zero-point energies of the three systems is about $12.3 \mathrm{eV}$ making it $1.3 \mathrm{eV}$ higher than was the case for PBE.

The local magnetization, shown in Table II, shows that, just like the HSE06 case, the correct local magnetization is obtained, even for the $S_{z}=1$ spin system, showing an important improvement over the normal PBE calculations.

The electronic band structures along the lines of high symmetry are presented in Fig. 7. Comparison to the HSE06 band structure ( $c f$. Fig. 3) shows a very good qualitative agreement already at first glance. At this point it is interesting to note that all band-structure related fitting variables are located on the $\Gamma$ - $\mathrm{R}$ high symmetry line. The fact that also the other high symmetry
TABLE V. Comparison PBE+U and PBE to HSE06.

\begin{tabular}{l|rrrrr}
\hline \hline variable & $\begin{array}{r}\text { HSE06 } \\
(\mathrm{eV})\end{array}$ & $\begin{array}{r}\mathrm{PBE}+\mathrm{U} \\
(\mathrm{eV})\end{array}$ & $\begin{array}{r}\Delta \\
(\mathrm{meV})\end{array}$ & $\begin{array}{r}\mathrm{PBE} \\
(\mathrm{eV})\end{array}$ & $\begin{array}{r}\Delta \\
(\mathrm{meV})\end{array}$ \\
\hline$\Delta_{0,1}$ & 0.141 & 0.153 & 12 & 0.079 & -62 \\
$\Delta_{0,2}$ & 1.461 & 1.096 & -365 & 1.696 & 235 \\
$\Delta^{\Gamma, 0}$ & $5.47^{a}$ & 5.502 & 32 & 4.930 & -540 \\
$\Delta_{1,2}^{\Gamma, 0}$ & -2.044 & -1.727 & 317 & -0.765 & 1279 \\
$\Delta_{1,3}^{\Gamma, 0}$ & -2.813 & -2.726 & 87 & -1.129 & 1684 \\
$\Delta_{2,2^{\prime}, 0}^{R^{\prime}}$ & 0.000 & -0.054 & -54 & 0.142 & 142 \\
$\Delta^{\Gamma, 1}$ & $5.47^{a}$ & 5.547 & 77 & 5.081 & -389 \\
$\Delta_{1,3}^{\Gamma, 1}$ & -1.691 & -1.506 & 185 & 0.090 & 1781 \\
$\Delta_{3,1}^{R^{\prime}, 1}$ & 0.185 & 0.310 & 125 & -0.709 & -894 \\
$\Delta^{\Gamma, 2}$ & $5.47^{a}$ & 6.135 & 665 & 5.110 & -360 \\
$\Delta_{1,1}^{\Gamma, 2}$ & -3.366 & -3.099 & 267 & -1.672 & 1694 \\
$\Delta_{1,2}^{\Gamma, 0^{*}}$ & 0.340 & 0.474 & 134 & 1.569 & 1229 \\
$\Delta_{1,0^{\prime}, 2}^{R^{\prime}}$ & -0.896 & -0.779 & 117 & 0.617 & 1513 \\
$\Delta_{3,0^{*}}^{R^{\prime}, 2}$ & -0.264 & -0.157 & 107 & 1.145 & 1409 \\
\hline \hline
\end{tabular}

${ }^{a}$ The experimental band gap was chosen instead of the HSE06 band gap.

lines $(\Gamma-\mathrm{X}, \Gamma-\mathrm{M}$, and $\mathrm{M}-\mathrm{X})$ show very good agreement supports the robustness of the $(\mathrm{U}, \mathrm{J})$-parameter pair.

Each of the defect states is located at the position they are expected, showing the correct order of $\uparrow$ and $\downarrow$ states. The host band gap is smaller than is the case for HSE06, but this is as expected as we fitted against the experimental band gap, which is almost one electronvolt smaller than the HSE06 one. This difference is mainly visible in the host valence band being closer to the Fermi level. Table $\mathrm{V}$ presents a comparison of the PBE and $\mathrm{PBE}+\mathrm{U}$ values of the selected fitting variables to the reference HSE06 values. The differences with the HSE06 values are indicated as $\Delta$. The $\mathrm{PBE}+\mathrm{U}$ values present a clear improvement over the PBE case. For the $S_{z}=0$ system, which already shows a good qualitative picture (cf Fig. 2), the separation between the defect states in the band gap are improved quantitatively by $1 \mathrm{eV}$ at the $\Gamma$-point. Also the small shape difference seen along the $\mathrm{R}-\Gamma$ line is entirely removed. For the $S_{z}=1$ system, the highest $\uparrow$ band, which in PBE is straddling the Fermi level, moves up to above the Fermi level but remains below the lowest in-gap $\downarrow$ band. In case of the $S_{z}=2$ system the large deviations seen for PBE are reduced by an order of magnitude, and the $\downarrow$ defect state at the valence band edge moves up to the Fermi level showing the expected crossing of the $\uparrow$ and $\downarrow$ states.

\section{The $\langle 001\rangle$ split-interstitial defect}

As a limited test of the transferability of the fitted $\mathrm{U}$ and $\mathrm{J}$ parameters, we considered the $\langle 001\rangle$ split-interstitial defect. ${ }^{36-40}$ The choice of this defect is threefold. Firstly, as for the vacancy defect, this 

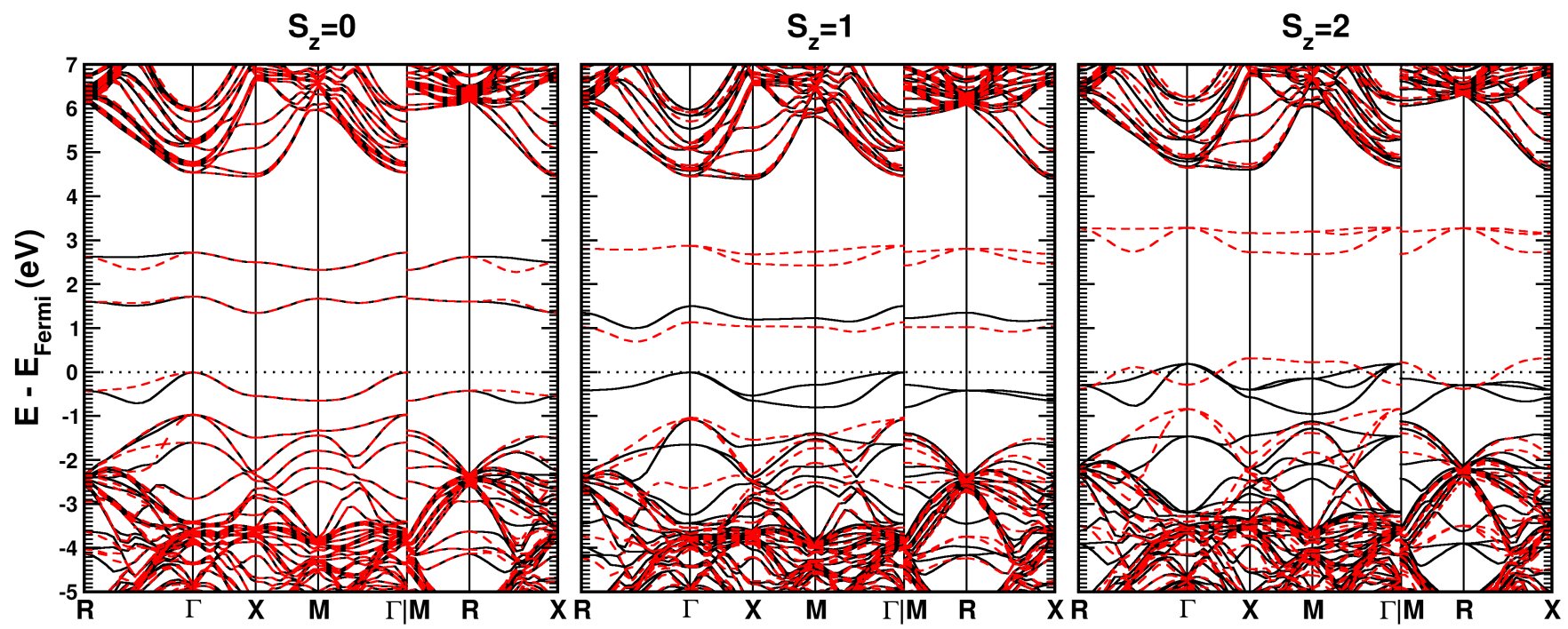

FIG. 7. PBE+U electronic band structure for a single C-vacancy defect in a 64-atom super cell. Majority/minority bands are show as solid/dashed curves. The DFT $+\mathrm{U}$ parameters are chosen as $\mathrm{U}=8.56 \mathrm{eV}$ and $\mathrm{J}=15.06 \mathrm{eV}$.

defect system only contains C atoms. Secondly, in contrast to the vacancy defect, the defect states for the split-interstitial are qualitatively well described at the DFT level, as such, it provides a testcase indicating whether good results are not deteriorated. This last aspect is important when studying defect complexes containing different contributing defects (e.g. the $\langle 001\rangle$ split-interstitial compressed vacancy defect $\left.{ }^{36}\right)$. Thirdly, the split-interstitial has been linked to the R2 defect, which is a fundamental damage product with a high production rate. ${ }^{36,37}$

The computational settings used for this defect are the same as those used for the vacancy defect. A conventional cubic 64-atom diamond super cell is modified to include one additional $\mathrm{C}$ atom. The second $\mathrm{C}$ atom of the split-interstitial defect consists of the nearby $\mathrm{C}$ atom displaced along the $\langle 001\rangle$ direction from its original position. The structure is then optimized using the PBE functional. As the use of $\mathrm{U}$ and $\mathrm{J}$ parameters has a strong influence on the lattice parameter, as shown in the previous section, and our goal is mainly to correct the electronic structure, we opt not to use the DFT $+U$ framework during the structure optimization. The resulting optimized structure is then used to calculate the electronic band structure with the PBE, HSE06, and $\mathrm{PBE}+\mathrm{U}$ functionals. Note that no new fitting of the $\mathrm{U}$ and $\mathrm{J}$ parameters was performed.

Both $\mathrm{C}$ atoms of the split-interstitial defect are $s p^{2}$ bonded, with one non-bonding $p_{\pi}$ electron remaining each. As a result, in addition to the closed shell (cs) configuration, two possible spin configurations are possible: (1) a singlet state, $S_{z}=0$, with the two $p_{\pi}$ electrons anti-parallel and (2) a triplet state, $S_{z}=1$, with both $p_{\pi}$ electrons parallel. In this section, we will consider all three spin-configurations as they lead to very distinct configurations of gap-states.
TABLE VI. Properties of the split-interstitial defect: the formation energy, $E_{f}$, the relative stability with regard to the $S_{z}=0$ ground state, $\Delta_{S_{z}=0}$, and band gap features ( $c f$. Fig. 8 . All energies are given in $\mathrm{eV}$.

\begin{tabular}{|c|c|c|c|}
\hline & HSE06 & $\mathrm{PBE}$ & $\mathrm{PBE}+\mathrm{U}$ \\
\hline & \multicolumn{3}{|c|}{$S_{z}=0$} \\
\hline$\overline{E_{f}}$ & 10.534 & 10.801 & 11.252 \\
\hline$\Delta^{\Gamma, 0}$ & 5.429 & 4.222 & 4.626 \\
\hline$\Delta_{v, 1}^{\Gamma, 0}$ & 0.990 & 1.198 & 0.551 \\
\hline$\Delta_{v, 2}^{\Gamma, 0}$ & 4.032 & 2.660 & 3.605 \\
\hline \multirow[t]{2}{*}{$\Delta_{2, c}^{M, 0}$} & 0.671 & 0.980 & 0.593 \\
\hline & \multicolumn{3}{|c|}{$S_{z}=1$} \\
\hline$\overline{E_{f}}$ & 10.558 & 10.822 & $\overline{11.289}$ \\
\hline$\Delta_{S_{z}=0}$ & 0.024 & 0.021 & 0.037 \\
\hline$\Delta^{\Gamma, 1}$ & 5.467 & 4.249 & 4.701 \\
\hline$\Delta_{v, 1}^{\Gamma, 1}$ & 1.056 & 1.244 & 0.639 \\
\hline$\Delta_{v, 2}^{\Gamma, 1}$ & 4.072 & 2.696 & 3.668 \\
\hline$\Delta_{1,1^{\prime}}^{X, 1}$ & 0.552 & 0.538 & 0.477 \\
\hline$\Delta_{2^{\prime}, c}^{X, 1}$ & 0.676 & 0.954 & 0.602 \\
\hline & \multicolumn{3}{|c|}{ closed shell } \\
\hline$\overline{E_{f}}$ & 11.920 & 11.367 & 12.752 \\
\hline$\Delta_{S_{z}=0}$ & 1.386 & 0.566 & 1.500 \\
\hline$\Delta^{\Gamma, c s}$ & 5.462 & 4.252 & 4.690 \\
\hline$\Delta_{v, 1}^{\Gamma, c s}$ & 2.441 & 1.837 & 1.925 \\
\hline$\Delta_{1,1^{\prime}}^{X^{\prime}, c s}$ & 0.629 & 0.580 & 0.476 \\
\hline$\Delta_{1^{\prime}, c}^{X^{\prime}, c s}$ & 2.412 & 1.893 & 2.495 \\
\hline
\end{tabular}

In Fig. 8 the HSE06 electronic band structure for different spin configurations of the split-interstitial defect are shown. This picture does not change qualitatively when using the PBE and $\mathrm{PBE}+\mathrm{U}$ functional, in contrast to what we saw for the vacancy defect.

Calculated properties of the split-interstitial defect are presented in Table VI. These results show that this de- 

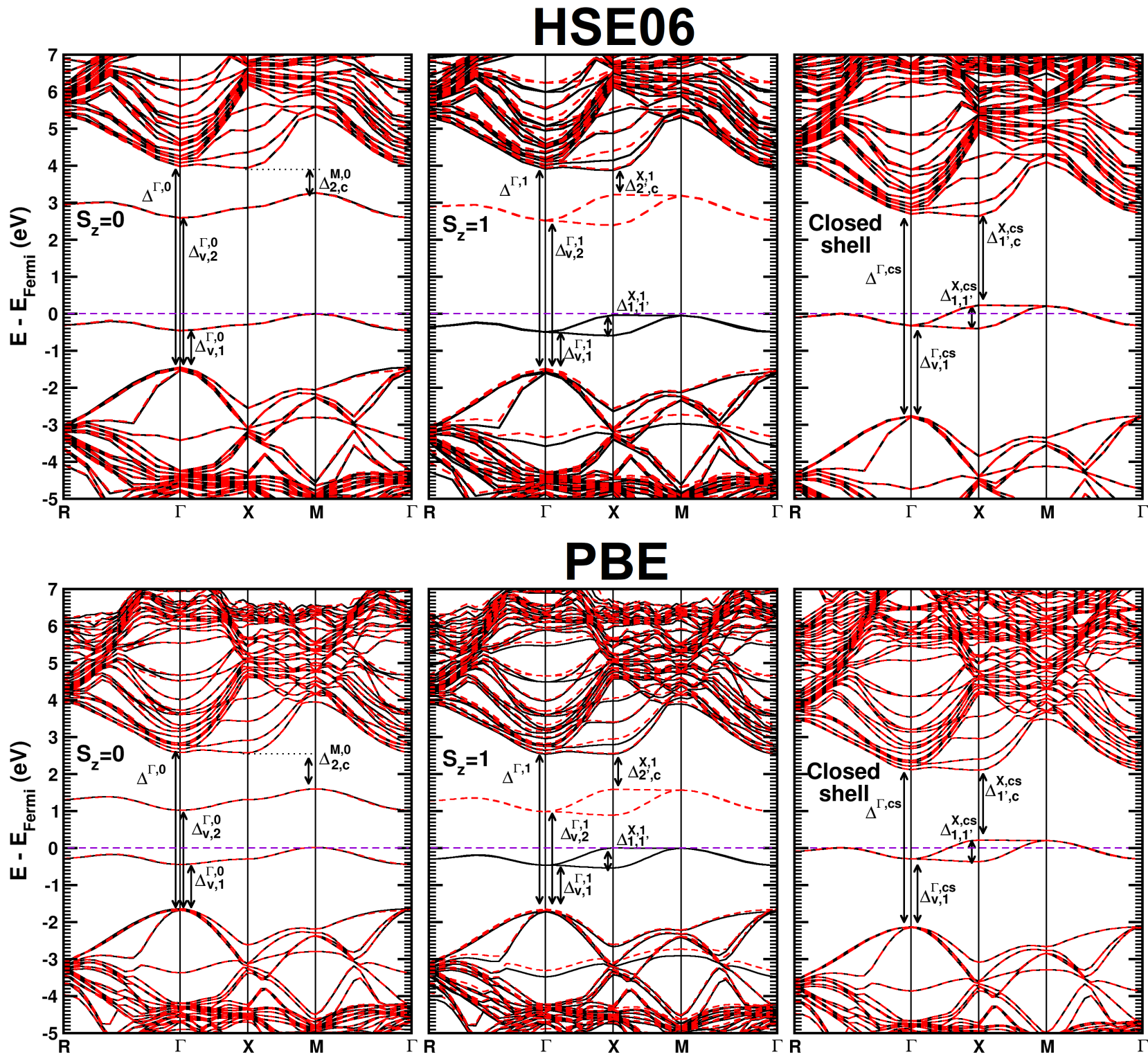

PBE+U
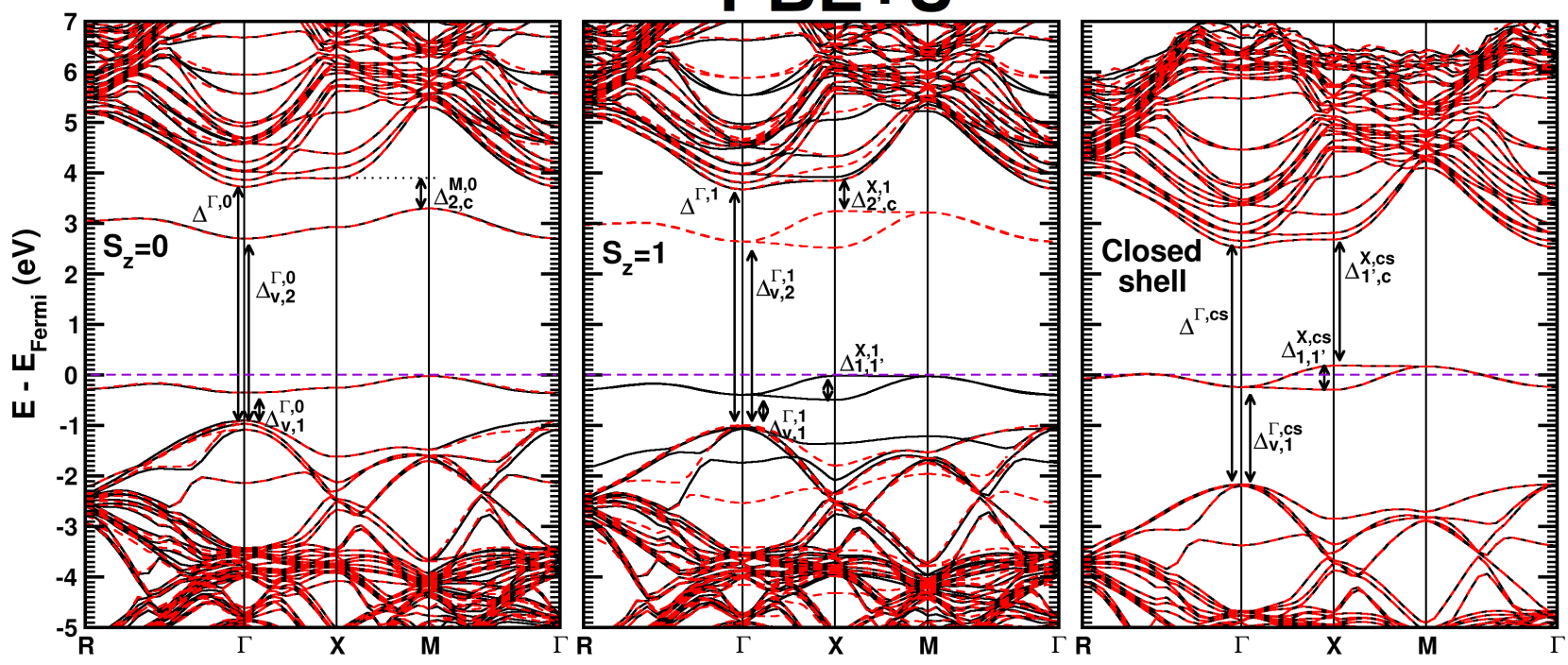

FIG. 8. The electronic band structure for the split-interstitial defect in a 65-atom super cell, using different functionals (top: HSE06, middle: PBE and bottom: PBE+U). Majority/minority bands are show as solid/dashed curves. The first Brillouin zone and the lines of high symmetry are shown in Fig. 1b. The $U$ and $J$ parameters used for the PBE+U calculation are those obtained from the neutral vacancy system: $\mathrm{U}=8.56 \mathrm{eV}$ and $\mathrm{J}=15.06 \mathrm{eV}$. 
fect is already qualitatively well described using the PBE functional, when comparing the HSE06 results. Looking at the $\mathrm{PBE}+\mathrm{U}$ results obtained for $(\mathrm{U}, \mathrm{J})=(8.56,15.06)$ $\mathrm{eV}$, we note that most features show improvement over the PBE results. The relative stability of the closed shell configuration is significantly overestimated by the $\mathrm{PBE}$ functional, while for the $\mathrm{PBE}+\mathrm{U}$ functional a relative stability comparable to the HSE06 value calculated in this work and B3LYP values in literature is found. ${ }^{40} \mathrm{It}$ is interesting to note that for this specific defect, the HSE06 functional presents the experimentally observed bulk band gap $\left(\Delta^{\Gamma, x}, \mathrm{x}=0,1, \mathrm{cs}\right)$ for diamond. PBE, as expected, significantly underestimates this gap, while the $\mathrm{PBE}+\mathrm{U}$ results improve the situation but not entirely. This may be related to the fact that we did not fit to the (overestimated) HSE06 band gap for the vacancy defect (which would have been detrimental for the vacancy defect). When looking at the positions of the defect states, we notice that in comparison to the HSE06 results, the PBE calculations place the defect states further from the band gap edges. This error is partially resolved by the $\mathrm{PBE}+\mathrm{U}$ functional, with the limitation that the $\mathrm{PBE}+\mathrm{U}$ band gap is still about 0.8 $\mathrm{eV}$ smaller than the HSE06 band gap.

\section{CONCLUSION}

In this work, we revisited the neutral C-vacancy using the PBE and HSE06 functionals. We show that for PBE the correct stability order is closely related to the correct local magnetization of the carbon atoms neighboring the vacancy. The latter was shown to depend significantly on the initial guess, indicative of a phase space with many local minima, making this an important aspect to consider when simulating defects in diamond. We showed that even if the stability order is correctly predicted, the defect states in the band gap are not qualitatively correct for the $S_{z}=1$ and 2 spin states.

Although a qualitatively and quantitatively more accurate picture can be obtained using hybrid functionals, their prohibitively high computational cost makes them impractical for large scale use. ${ }^{22}$ To alleviate this problem, we propose the use of the DFT $+\mathrm{U}$ methodology using the rotationally invariant implementation as proposed by Liechtenstein and co-workers. The on-site Coulomb and exchange parameters $\mathrm{U}$ and $\mathrm{J}$ are obtained through a fitting procedure taking into account the specific features of the electronic structure of the defect states of the three spin configurations of the neutral $\mathrm{C}$ vacancy defect. Using $(\mathrm{U}, \mathrm{J})=(8.56,15.06) \mathrm{eV}$, the electronic structure of the C-vacancy is calculated and compared to HSE06 reference results. The DFT $+\mathrm{U}$ results were shown to accurately reproduce the order and position of the defect states. In addition, the $\mathrm{DFT}+\mathrm{U}$ results also presented the correct stability order and local magnetization of the neighboring $\mathrm{C}$ atoms.

In addition, we showed that the proposed $\mathrm{PBE}+\mathrm{U}$ approach will improve, to some extend, the electronic structure of the $\langle 001\rangle$ split-interstitial defect. However, as the latter is already qualitatively well described by the PBE functional, the gain is much less significant than for the vacancy defect.

The presented $(\mathrm{U}, \mathrm{J})$ pair should be well suited for the investigation of vacancy based defects, such as the neutral vacancy considered in this work (at the same or even lower concentration), but even defect complexes may benefit from this approach. The description of dopant defects might be improved as well, but this will strongly depend on the nature of the dopant ( $d$ - and $f$-block dopants may not be properly described), and is a subject of future work. These results show that $\mathrm{DFT}+\mathrm{U}$ can be a computationally cheap (i.e. $100 \times$ to $1000 \times$ faster) alternative to hybrid functional calculations, using plane wave basis sets, for the study of defects in diamond in large scale studies.

\section{PRIME NOVELTY STATEMENT}

$\mathrm{DFT}+\mathrm{U}$ can be used to provide hybrid functional quality electronic structure results at a fraction of the cost. For the neutral vacancy defect in diamond the optimum parameter-pair is $(\mathrm{U}, \mathrm{J})=(8.56,15.06) \mathrm{eV}$. The same parameters are also suitable for other intrinsic defects such as the $\langle 001\rangle$ split-interstitial defect.

\section{ACKNOWLEDGMENTS}

The financial support via the Methusalem project NANO is greatly appreciated. The computational resources and services used in this work were provided by the VSC (Flemish Supercomputer Center), funded by the Research Foundation - Flanders (FWO) and the Flemish Government - department EWI. DEPV is a postdoctoral researcher funded by the Research Foundation - Flanders (FWO) (project no. 12S3415N).
* Danny.vanpoucke@UHasselt.be

1 Andrew Magyar, Wenhao Hu, Toby Shanley, Michael E. Flatté, Evelyn $\mathrm{Hu}$, and Igor Aharonovich, "Synthesis of luminescent europium defects in diamond," Nat. Commun. 5, 3523 (2014).
2 Jakub Cajzl, Pavla Nekvindova, Anna Mackova, Petr Malinsky, David Sedmidubsky, Michal Husak, Zdenek Remes, Marian Varga, Alexander Kromka, Roman Bottger, and Jiri Oswald, "Erbium ion implantation into diamond measurement and modelling of the crystal structure," 
Phys. Chem. Chem. Phys. 19, 6233-6245 (2017)

3 N. A. Katcho, J. Carrete, Wu Li, and N. Mingo, "Effect of nitrogen and vacancy defects on the thermal conductivity of diamond: An ab initio Green's function approach," Phys. Rev. B 90, 094117 (2014).

${ }^{4}$ R. P. Messmer and G. D. Watkins, "Molecular-Orbital Treatment for Deep Levels in Semiconductors: Substitutional Nitrogen and the Lattice Vacancy in Diamond," Phys. Rev. B 7, 2568-2590 (1973).

5 J. Bernholc, A. Antonelli, T. M. Del Sole, Y. Bar-Yam, and S. T. Pantelides, "Mechanism of self-diffusion in diamond," Phys. Rev. Lett. 61, 2689-2692 (1988).

${ }^{6}$ S. J. Breuer and P. R. Briddon, "Ab initio investigation of the native defects in diamond and self-diffusion," Phys. Rev. B 51, 6984-6994 (1995).

7 Lin Hong Li and J. E. Lowther, "Lattice relaxation at vacancy aggregates in diamond," Phys. Rev. B 53, 1127711280 (1996).

8 A. Zywietz, J. Furthmüller, and F. Bechstedt, "Neutral Vacancies in Group-IV Semiconductors," phys. status solidi B 210, 13-29 (1998).

9 A. Zywietz, J. Furthmüller, and F. Bechstedt, "Spin state of vacancies: From magnetic Jahn-Teller distortions to multiplets," Phys. Rev. B 62, 6854-6857 (2000).

10 Randolph Q. Hood, P. R. C. Kent, R. J. Needs, and P. R. Briddon, "Quantum Monte Carlo Study of the Optical and Diffusive Properties of the Vacancy Defect in Diamond," Phys. Rev. Lett. 91, 076403 (2003).

11 Jihye Shim, Eok-Kyun Lee, Y. J. Lee, and R. M. Nieminen, "Density-functional calculations of defect formation energies using supercell methods: Defects in diamond," Phys. Rev. B 71, 035206 (2005).

12 Istvan Laszlo, Miklos Kertesz, Brad Slepetz, and Yury Gogotsi, "Simulations of large multi-atom vacancies in diamond," Diam. Relat. Mater. 19, 1153-1162 (2010).

13 David Hyde-Volpe, Brad Slepetz, and Miklos Kertesz, "The $[\mathrm{V}-\mathrm{C}=\mathrm{C}-\mathrm{V}]$ Divacancy and the Interstitial Defect in Diamond: Vibrational Properties," J. Phys. Chem. C 114, 9563-9567 (2010).

14 Alessandro Zelferino, Simone Salustro, Jacopo Baima, Valentina Lacivita, Roberto Orlando, and Roberto Dovesi, "The electronic states of the neutral vacancy in diamond: a quantum mechanical approach," Theor. Chem. Acc. 135, 74 (2016).

15 Jacopo Baima, Alessandro Zelferino, Paolo Olivero, Alessandro Erba, and Roberto Dovesi, "Raman spectroscopic features of the neutral vacancy in diamond from ab initio quantum-mechanical calculations," Phys. Chem. Chem. Phys. 18, 1961-1968 (2016).

16 Christian Kranert, Chris Sturm, Rdiger Schmidt-Grund, and Marius Grundmann, "Raman tensor elements of $\beta \mathrm{Ga}_{2} \mathrm{O}_{3}$," Sci. Rep. 6, 35964 (2016).

17 Adam Gali, Erik Janzén, Péter Deák, Georg Kresse, and Efthimios Kaxiras, "Theory of Spin-Conserving Excitation of the $N-V^{-}$Center in Diamond," Phys. Rev. Lett. 103, 186404 (2009).

18 Thomas M. Henderson, Joachim Paier, and Gustavo E. Scuseria, "Accurate treatment of solids with the HSE screened hybrid," Phys. status solidi B 248, 767-774 (2011)

19 Peter Deák, Bálint Aradi, Moloud Kaviani, Thomas Frauenheim, and Adam Gali, "Formation of NV centers in diamond: A theoretical study based on calculated transitions and migration of nitrogen and vacancy related de- fects," Phys. Rev. B 89, 075203 (2014).

20 Roman Ovcharenko, Elena Voloshina, and Joachim Sauer, "Water adsorption and O-defect formation on $\mathrm{Fe}_{2} \mathrm{O}_{3}(0001)$ surfaces," Phys. Chem. Chem. Phys. 18, 25560-25568 (2016).

21 Danny E. P. Vanpoucke, "Linker Functionalization in MIL47(V)-R MetalOrganic Frameworks: Understanding the Electronic Structure," J. Phys. Chem. C 121, 8014-8022 (2017).

22 The high computational cost for hybrid functionals in solids is due to several aspects. The basis-set size being the most obvious one, as more basis functions means more overlap integrals needing to be calculated. In this regard, the use of a localized basis set reduces the overall cost significantly, this due to the much smaller size of the basis set, on the one hand, and the short range nature of localized basis sets, which implicitly truncates the non-local part of the functional, on the other. A second aspect which plays an important role is the integration of the first Brillouin zone. As the Fock-exchange is calculated as a double sum over all basis-functions and all k-points, the computational cost is expected to increase with the square of the k-points used. As a result, reducing the k-point grid of the non-local part of the functional to the $\Gamma$-point only will give rise to a tremendous speedup. As such, hybrid calculations on the system at hand are merely a factor of 7 more expensive than PBE. The accuracy, however, is entirely lost.

23 C. A. Coulson and Mary J. Kearsley, "Colour Centres in Irradiated Diamonds. I," Proc. R. Soc. Lond. A 241, 433454 (1957).

24 F. Jelezko and J. Wrachtrup, "Single defect centres in diamond: A review," Phys. Status Solidi A 203, 3207-3225 (2006).

25 Marcus W. Doherty, Neil B. Manson, Paul Delaney, Fedor Jelezko, Joerg Wrachtrup, and Lloyd C. L. Hollenberg, "The nitrogen-vacancy colour centre in diamond," Phys. Rep.-Rev. Sec. Phys. Lett. 528, 1-45 (2013).

26 Elke Neu, Christian Hepp, Michael Hauschild, Stefan Gsell, Martin Fischer, Hadwig Sternschulte, Doris Steinmueller-Nethl, Matthias Schreck, and Christoph Becher, "Low-temperature investigations of single silicon vacancy colour centres in diamond," New J. Phys. 15 (2013).

27 Christian Hepp, Tina Mueller, Victor Waselowski, Jonas N. Becker, Benjamin Pingault, Hadwig Sternschulte, Doris Steinmueller-Nethl, Adam Gali, Jeronimo R. Maze, Mete Atatuere, and Christoph Becher, "Electronic Structure of the Silicon Vacancy Color Center in Diamond," Phys. Rev. Lett. 112 (2014).

28 Alejandro J. Garza and Gustavo E. Scuseria, "Predicting Band Gaps with Hybrid Density Functionals," J. Phys. Chem. Lett. 7, 4165-4170 (2016).

29 Kevin Hendrickx, Danny E. P. Vanpoucke, Karen Leus, Kurt Lejaeghere, Andy Van Yperen-De Deyne, Veronique Van Speybroeck, Pascal Van Der Voort, and Karen Hemelsoet, "Understanding Intrinsic Light Absorption Properties of UiO-66 Frameworks: A Combined Theoretical and Experimental Study," Inorg. Chem. 54, 1070110710 (2015).

${ }^{30}$ K. Czelej, P. Śpiewak, and K. J. Kurzydłowski, "Electronic structure of substitutionally doped diamond: Spinpolarized, hybrid density functional theory analysis," Diam. Rel. Mater. 75, 146-151 (2017). 
31 A Mainwood, J E Lowther, and J A Van Wyk, "The R2 EPR centre and $1.685 \mathrm{eV}$ absorption line in diamond," J. Phys. Condens. Matter 5, 7929 (1993).

32 Danny E. P. Vanpoucke, Patrick Bultinck, Stefaan Cottenier, Veronique Van Speybroeck, and Isabel Van Driessche, "Aliovalent doping of $\mathrm{CeO}_{2}$ : DFT study of oxidation state and vacancy effects," J. Mater. Chem. A 2, 1372313737 (2014).

33 Class Persson and Susanne Mirbt, "Improved Electronic Structure and Optical Properties of sp-Hybridized Semiconductors Using LDA $+\mathrm{U}^{S I C}$," Braz. J. Phys. 36, 286-290 (2006).

34 A. I. Liechtenstein, V. I. Anisimov, and J. Zaanen, "Density-functional theory and strong interactions: Orbital ordering in Mott-Hubbard insulators," Phys. Rev. B 52, R5467-R5470 (1995).

35 S. L. Dudarev, G. A. Botton, S. Y. Savrasov, C. J. Humphreys, and A. P. Sutton, "Electron-energy-loss spectra and the structural stability of nickel oxide: An LSDA+U study," Phys. Rev. B 57, 1505-1509 (1998).
${ }^{36}$ D. C. Hunt, D. J. Twitchen, M. E. Newton, J. M. Baker, T. R. Anthony, W. F. Banholzer, and S. S. Vagarali, "Identification of the neutral carbon 100-split interstitial in diamond," Phys. Rev. B 61, 3863-3876 (2000).

37 Gordon Davies, Hannah Smith, and H. Kanda, "Selfinterstitial in diamond," Phys. Rev. B 62, 1528-1531 (2000).

38 J. P. Goss, B. J. Coomer, R. Jones, T. D. Shaw, P. R. Briddon, M. Rayson, and S. Öberg, "Self-interstitial aggregation in diamond," Phys. Rev. B 63, 195208 (2001).

39 Hannah E. Smith, Gordon Davies, M. E. Newton, and H. Kanda, "Structure of the self-interstitial in diamond," Phys. Rev. B 69, 045203 (2004).

40 Simone Salustro, Alessandro Erba, Claudio M. ZicovichWilson, Yves Noel, Lorenzo Maschio, and Roberto Dovesi, "Infrared and Raman spectroscopic features of the selfinterstitial defect in diamond from exact-exchange hybrid DFT calculations," Phys. Chem. Chem. Phys. 18, 2128821295 (2016). 